\title{
Les défis de la progression des cancers en Afrique
}

\author{
A. Ly \\ (C) Springer-Verlag 2009
}

Les perspectives épidémiologiques des cancers qui se profilent d'ici à l'horizon 2020 prévoient une progression continue en termes d'incidence et de mortalité. Si cet impact croissant des pathologies tumorales est individuellement et collectivement à déplorer à l'échelle mondiale, il a davantage de conséquences néfastes dans les pays en développement sur lesquels pèseront $72 \%$ du fardeau global de la maladie.

En raison de multiples facteurs agissant en synergie qui vont de l'explosion démographique à la pression des maladies infectieuses et aux méfaits du tabagisme, des déséquilibres alimentaires aux pollutions environnementales et aux prédispositions ou susceptibilités génétiques, le nombre d'Africains et d'Africaines exposés aux risques de cancer croîtra de façon inéluctable. En effet, compte tenu des tendances actuelles, un doublement de l'incidence des cancers est prévu en Afrique, soit près d'un million de nouveaux cas tous sexes confondus selon les organisations internationales de santé.

En se fondant sur ces chiffres et sur de nombreuses observations cliniques concordantes, il est permis de battre en brèche les préjugés et apriorismes qui euphémisent la gravité de la maladie sur le continent africain en la restreignant aux seuls pays du nord.

Au contraire, l'analyse lucide de la situation épidémiologique montre que les maladies non transmissibles se sont durablement installées dans les populations africaines au même titre que les maladies transmissibles. Certaines, telles que les hépatites $\mathrm{B}$ et $\mathrm{C}$, le sida, les infections à papillomavirus humains ou à Helicobacter pilori, par leur chronicité, contribuent d'ailleurs à l'expansion des néoplasies en Afrique. Il est hors de propos, ici, d'établir des rapports conflictuels ou hiérarchiques même implicites entre ces diverses pathologies qui, sur le sol africain, sont toutes sources de morts et de souffrances humaines.

Le déclin espéré des maladies infectieuses au cours du siècle dernier, qui devait engendrer une transition épidémiologique conduisant principalement aux maladies non transmissibles chroniques, n'a pas eu lieu. Les deux types de maladies, transmissibles et non transmissibles, coexistent, se

A. Ly $(\bowtie)$

Afrocancer AC, BP 60751, F-75827 Paris cedex 17, France

e-mail : adamaly@gmail.com développent et entretiennent des liens scientifiquement prouvés depuis longtemps. Ainsi, 25 à $30 \%$ des cancers en Afrique ont une étiologie infectieuse.

Il est aussi établi que le sarcome de Kaposi et les lymphomes non hodgkiniens sont, en fréquence, les tumeurs malignes dont souffrent le plus les malades infectés par le VIH/sida.

En outre, les régions infestées par le Plasmodium falciparum, agent causal du paludisme, sont celles où est prévalent de façon exagérée le lymphome de Burkitt, un sous-type de lymphome non hodgkinien dont le développement oncogénétique est la résultante d'une co-infection avec le virus d'Epstein-Barr. De même, la bilharziose, autre maladie parasitaire endémique des régions africaines, induit, à l'état chronique, un carcinome épidermoïde de la vessie.

Ce sont là quelques caractéristiques qui déterminent la singularité des mécanismes cellulaires et moléculaires de l'oncogenèse et celle de la physiopathologie des néoplasies en zone tropicale et subtropicale sous l'influence de l'environnement microbiologique et parasitaire.

Par conséquent, c'est éviter un péril de truisme que de reconnaître que les cancers constituent un enjeu prioritaire africain de santé publique.

L'ampleur prévue de l'augmentation de l'incidence des cancers en Afrique questionne sur les mesures persistantes et conséquentes d'ordre médical, scientifique et social à prendre pour promouvoir davantage la lutte contre les cancers sur le continent.

Au-delà de la description et de l'analyse de ces problèmes cruciaux de santé et du contexte d'évolution progressive des maladies malignes en Afrique, de multiples défis sont à relever pour tendre vers un renversement de ces perspectives épidémiologiques redoutées et lourdes de menaces et de conséquences pour les populations africaines.

Il est urgent, à court terme, de consacrer des efforts massifs à la définition de stratégies et de programmes africains d'envergure de lutte contre le cancer, et de renforcer les systèmes de santé qui en sont les piliers.

Ainsi, il nous a paru opportun de créer une revue scientifique et médicale entièrement dédiée à cette problématique des affections tumorales en Afrique : le Journal africain du cancer (JAC)/African journal of cancer (AJC). 
Le $J A C$ est un trimestriel créé sous l'égide d'Afrocancer, une association internationale de lutte contre le cancer, qui se veut, d'une part, un porte-parole de cette lutte longue et ardue et, d'autre part, un lieu d'expression des divers acteurs du combat contre les cancers.

Lancé dans le sillage du livre "Le cancer en Afrique : de l'épidémiologie aux applications et perspectives de la recherche biomédicale », ouvrage collectif et multidisciplinaire édité par l'Institut national du cancer (INCa), le JAC sera un journal d'information et de formation continue qui, en plus de la diffusion d'articles originaux, de revues générales et de synthèse respectant les règles internationales quant à leur fond et leur forme, veut susciter chez les jeunes universitaires un intérêt pour l'oncologie.

À travers la sélection d'articles publiée et les thèmes abordés, le JAC s'installera comme une revue médicale de qualité, innovante, intégrant prioritairement la réflexion transversale et reflétant à la fois les avancées de la recherche biomédicale au plan continental et international. Au niveau transversal, les contributions des économistes de la santé, des sociologues, des psychologues et des juristes seront encouragées.

Par ailleurs, un des axes focaux du JAC sera de faciliter les échanges avec la communauté scientifique internationale en montrant autant les progrès que les insuccès ou les obstacles que rencontre la pratique de l'oncologie clinique et fondamentale en Afrique.

De même, pour mener à bien ce rôle d'interface, nous serons les témoins avertis des nouveautés, des tentatives et essais mais aussi des échecs et limites des actions et programmes anticancéreux en cours dans les pays les plus avancés dans le combat contre le cancer.

En tout état de cause, notre souci permanent est que ces informations soient profitables aux professionnels de santé des pays africains engagés dans les diverses spécialités de la cancérologie, certes encore peu nombreux, eu égard à l'immensité de la tâche.

Parallèlement, un examen, sans fard, du contexte oncologique africain montre le nombre encore limité de sociétés savantes et surtout d'associations de bénévoles et d'acteurs de la société civile. Ce qui réduit l'impact des campagnes de prévention et de sensibilisation en direction du grand public. Cependant, le tissu associatif s'étoffe progressivement depuis quelques années sous la houlette des femmes, et c'est heureux puisqu'elles sont les premières victimes des néoplasmes en Afrique. Des actions de ce type et de nombreuses autres, venant des milieux hospitalouniversitaires, associatifs et humanitaires, jugées pertinentes, pourront être mises en évidence dans le JAC. C'est, en effet, une partie importante de la solution nécessitant des ressources humaines, des moyens financiers et d'outils matériels et pédagogiques pour freiner l'extension du fléau carcinologique en zone subsaharienne.
Enfin et surtout, l'essor véritable de la lutte contre le cancer en Afrique, de la recherche à la clinique et aux organisations sociales, réside dans la prise de mesures efficientes qui doivent être sous-tendues par une forte volonté politique. Nous en appellerons donc dans nos pages à un sens de l'engagement et à une éthique de la responsabilité des spécialistes, des politiques et des leaders d'opinions pour élargir ensemble le champ des possibles. En effet, les données autant épidémiologiques qu'étiologiques montrent que cette pathologie est une menace majeure pour la santé des populations africaines.

L'organisation de structures collectives pour une prise en charge socialisée de l'accès aux produits de santé en lieu et place des stratégies individuelles ou familiales non exemptes de risque faciliterait autant le parcours de soins des malades que la prévention et le dépistage précoce de la maladie qui sont liés aux changements des comportements délétères des populations. De telles habitudes comportementales sont modulables. Elles ne perdurent qu'à cause de la pauvreté, de l'ignorance des facteurs de risque, des freins et des obstacles culturels.

Ce sont donc autant de défis qui font que le combat africain contre le cancer doit changer d'échelle. Ce sont aussi autant de raisons d'être du JAC.

Nous serons présents pour appréhender et accompagner ces mutations en cours et participer de manière active ou incitative à la mobilisation générale et à la recherche de solutions durables qu'elles soient biomédicales ou socioéconomiques, voire politiques.

Faire vivre un journal qui affiche cette prétention n'est pas chose aisée.

Aussi, je voudrais remercier également toute l'équipe de Springer, en particulier, son directeur, M. Guido ZosimoLandolfo, Mme Nathalie L'horset-Poulain, éditrice, responsable du pôle Oncologie et Mme Nathalie Huilleret, directrice éditoriale, qui se sont engagés courageusement à relever ce défi éditorial...

Nous sommes fiers et encouragés dans nos choix par l'enthousiasme suscité dans la communauté médicale et scientifique, africaine et internationale, par l'annonce $\mathrm{du}$ lancement de ce premier grand journal africain de cancérologie.

Je voudrais remercier les membres de nos comités, éditorial et scientifique, composés de personnalités éminentes du monde de la cancérologie pour leur implication spontanée au projet d'édition d'un journal africain du cancer.

Ces comités sont, à dessein, structurés de la sorte, constitués de personnalités de pays, de culture, de spécialités, de parcours de vie différents, parce que notre approche est résolument pluridisciplinaire, que notre credo est le partage des connaissances et des expériences, et que la lutte contre le cancer, sous toutes ses dimensions, doit ignorer tous types de frontières, de sectarisme et de limitations. 
Le professeur Jean Dausset, prix Nobel de médecine, dont les vœux de réussite nous accompagnent, aurait parlé de prise de « responsabilité scientifique et politique » pour reprendre le titre de la préface qu'il a rédigée pour le livre « Le cancer en Afrique ».

Enfin, nous espérons rencontrer l'adhésion et le soutien des professionnels de santé d'Afrique et d'ailleurs, notre lectorat, à qui ce journal est prioritairement destiné en espérant qu'ils contribueront par leur soumission d'articles en langues française ou anglaise, par leurs commentaires et par le nombre élevé de leurs abonnements à faire du $J A C / A J C$ une revue biomédicale de référence.

Ce premier numéro du $J A C$ présente des articles qui traitent des cancers du sein au Gabon, des caractéristiques épidémiologiques des hémopathies malignes à Abidjan en Côte-d'Ivoire, de l'épidémiologie du cancer du côlon dans
l'Ouest algérien et des mélanomes malins primitifs extracutanés à Brazzaville.

Une technique d'invalidation du gène de l'insuline like growth factor-1 (IGF-1), facteur de croissance des tumeurs, est l'objet des travaux de Trojan et al. L'importance des mycotoxines comme facteur de risque de cancers est abordée par Annie Pfohl-Leszkowicz tandis que le risque de survenue de tumeurs mammaires chez les Tunisiennes lié à la présence de certains allèles du CMH-1 est exploré par Baccar et al.

Du côté des associations, l'Alliance mondiale contre le cancer (AMCC) décrit ses actions passées et futures de lutte contre le cancer dans les pays en voie de développement.

En somme, ce numéro 1 du Journal Africain du Cancer est caractérisé par son éclectisme qui va des aspects fondamentaux et cliniques aux politiques de santé publique. 